\title{
ASSISTÊNCIA MATERNO-INFANTIL, TIPO DE SERVIÇO UTILIZADO E CARACTERÍSTICAS SOCIODEMOGRÁFICAS EM CIDADE DO INTERIOR DA PARAÍBA
}

\author{
MATERNAL AND CHILD CARE, SERVICE USED AND SOCIO-DEMOGRAPHIC \\ CHARACTERISTICS IN A CITY IN THE INNER STATE OF PARAÍBA, BRAZIL
}

\author{
Dixis Figueroa Pedraza ${ }^{a^{*}}$, Erika Araujo \\ adixisfigueroa@gmail.com, ${ }^{\circ}$ morgannaneves@ hotmail.com \\ *Universidade Estadual da Paraíba - Campina Grande (PB), Brasil
}

Data de recebimento: 06/04/2016

Data de aceite: 25/08/2016

\section{RESUMO}

Objetivo: Analisar a assistência pré-natal e ao parto/puerpério no atendimento público e privado, segundo características sociodemográficas maternas, na cidade de Campina Grande, Paraíba. Metodologia: Estudo transversal, desenvolvido na cidade de Campina Grande, Paraíba. Entrevistaram-se 738 mães de filhos menores de um ano, incluindo informaçôes sociodemográficas e da atenção ao pré-natal, ao parto e ao puerpério, inclusive sobre o tipo de serviço utilizado. A distribuição relacionada ao tipo de serviço utilizado no pré-natal e no parto/puerpério foi analisada segundo as caraterísticas sociodemográficas maternas. Resultados: A utilização do serviço público foi de $77 \%$ na assistência pré-natal e de $80,1 \%$ no caso do parto/puerpério entre os entrevistados. Verificou-se vantagem sistemática na utilizaçáo de serviços públicos de saúde durante o pré-natal e o parto/puerpério nas mulheres de menor escolaridade e nas de famílias beneficiadas pelo Programa Bolsa Família. Conclusáo: Os resultados sugerem que a maior utilização dos serviços públicos de saúde coexiste com desigualdades no uso de serviços entre a população atendida pelo SUS e a população beneficiária de planos de saúde. Assim, remete-se à necessidade de açóes de melhoria da assistência pré-natal e ao parto/puerpério, sobretudo pelo setor público, que atende a população de maior vulnerabilidade.

Palavras-chave: Atenção à saúde; cuidado pré-natal; saúde materno-infantil; qualidade da assistência à saúde; fatores socioeconômicos.

\section{ABSTRACT}

Objective: To analyze the prenatal and delivery/postpartum care in the city of Campina Grande, Paraíba (BR), considering maternal sociodemographic characteristics, according to the service used (public or private). Methods: Cross-sectional study developed in the city of Campina Grande, Paraíba. A total of 738 mothers of children under one year of age were interviewed, and information included sociodemographic status and prenatal, delivery and postpartum care and type of service used. The distribution related to the type of service used in the prenatal and delivery/postpartum was analyzed according to maternal sociodemographic characteristics. Results: The use of public services was $77 \%$ in prenatal care and $80.1 \%$ in cases of delivery/postpartum among these mothers. There was a systematic advantage in the use of public health services for prenatal and delivery/postpartum by women with less education and families benefited from the Programa Bolsa Família (Brazilian's "Family Allowance Program"). Conclusion: The results suggest that the increased use of public health services coexists with inequalities in the use of health services among population served by SUS (Public Health System) and populations assisted by private health plans. Thus, there is a need for actions to improve prenatal care and delivery/postpartum, especially by the public sector for populations of greater vulnerability.

Keywords: Health care; prenatal care; maternal and child health; health care quality; socioeconomic factors. 


\section{Introdução}

À mulher deve ser garantida a acessibilidade aos serviços de saúde, com açóes que integrem todos os níveis de atenção ${ }^{1}$. O Ministério da Saúde do Brasil recomenda que os serviços de atenção à saúde devem assegurar à mulher o direito ao planejamento reprodutivo e a atenção humanizada à gravidez, ao parto e ao puerpério ${ }^{2,3}$. Nesse sentido, o Programa de Humanizaçáo do Pré-Natal e Nascimento (PHPN) traz diretrizes sobre um modelo nacional de açóes assistenciais para assegurar a melhoria do acesso, da cobertura e da qualidade do acompanhamento pré-natal, da assistência ao parto e puerpério, tanto para a gestante quanto para o recém-nascido ${ }^{4,5}$.

Além da influência das caraterísticas de organização e das práticas dos serviços de saúde, a gravidez, o parto e o nascimento associam-se a fatores sociais, econômicos e demográficos ${ }^{6}$. Nessa conjuntura, a situação da saúde no Brasil é marcada pelas iniquidades e pelo privilégio dos grupos sociais mais favorecidos, constituindo-se, portanto, em alvo importante de políticas públicas $^{7,8}$. Ainda, indicam-se disparidades na oferta, utilização e qualidade dos serviços dependentes da fonte prestadora ${ }^{9-11}$.

O presente estudo teve como objetivo analisar a distribuição das máes/filhos, segundo características sociodemográficas maternas, de acordo com o tipo de serviço utilizado (público ou privado) na assistência pré-natal e ao parto/pós-parto, na cidade de Campina Grande, Paraíba.

\section{Métodos}

Este é um trabalho transversal que forma parte da pesquisa "Chamada Neonatal", desenvolvida pela Escola Nacional de Saúde Pública Sérgio Arouca (Ensp), da Fundaçáo Oswaldo Cruz (Fiocruz), na primeira etapa da campanha nacional de vacinação, no dia 12 de junho de 2010. A Chamada Nacional objetivou obter informaçóes sobre morbidade materna, morbidade infantil e sobre as açóes do pacto pela reduçáo da mortalidade infantil em uma amostra representativa de mães e crianças menores de um ano residentes em 256 municípios das regióes da Amazônia e Nordeste.

$\mathrm{O}$ método, incluindo o treinamento, os instrumentos de coleta dos dados, o controle de qualidade e as perdas amostrais, é descrito detalhadamente na publicação oficial: "Avaliação da atenção ao pré-natal, ao parto e aos menores de um ano na Amazônia Legal e no Nordeste, Brasil, 2010"12.

O cálculo do tamanho da amostra considerou prevalência esperada de $22 \%$ de alguma complicação grave durante o parto (indicador de morbidade materna grave), erro de $4 \%$ e grau de confiança de $95 \%$, resultando em n=412 para um desenho aleatório simples. Sendo o inquérito desenvolvido por conglomerado, com sorteio em dois estágios, o tamanho da amostra foi multiplicado pelo fator de correção de desenho $(\operatorname{deff}=1,5)$, o que resultou em uma amostra de 750 pares mãe/filho. No primeiro estágio, foram sorteados os postos de vacinaçáo; no segundo, definiu-se uma fração de sorteio para cada posto, para selecionar de forma sistemática a fila de vacinação.

A pesquisa trouxe estimativas para os dezessete estados, suas capitais e, ainda, estimativas para o conjunto de municípios do interior de cada estado, incluindo, portanto, desenho amostral para o município de Campina Grande. O tamanho amostral foi de 633 pares mãe/ filho, representando $55,5 \%$ das 1.141 crianças menores de um ano vacinadas no dia da campanha de vacinação.

Dirigido às mães de crianças menores de um ano, o questionário incluiu informaçôes sobre características das famílias; atenção pré-natal; atenção ao parto e ao puerpério. Neste estudo, utilizaram-se variáveis de caraterização sociodemográfica das mães: anos de estudo (ensino fundamental incompleto, ensino fundamental completo/médio incompleto e ensino médio completo/superior); raça (branca, parda, negra, indígena/amarela); chefe de família mulher (sim/não); benefício do Programa Bolsa Família (sim/não). Como características da assistência ao pré-natal, ao parto e puerpério, considerou-se o tipo de serviço utilizado, se público ou privado.

Os questionários preenchidos foram digitalizados utilizando a tecnologia de construção de banco de dados e de imagens intelligent character recognition, analisando a consistência dos dados para cada questão. Valores não plausíveis foram conferidos com os valores registrados, utilizando o banco de imagens dos questionários.

A distribuição relacionada ao tipo de serviço utilizado foi analisada segundo as caraterísticas sociodemográficas maternas. $\mathrm{Na}$ abordagem analítica foi realizada análise bivariada, utilizando o teste de qui-quadrado de Pearson. Considerou-se um nível de significância $\mathrm{p}<0,05$. Utilizou-se o software SPSS versão 16 (PASW Inc. Chicago, Estados Unidos) para analisar os dados.

A pesquisa foi previamente aprovada pelo Comitê de Ética em Pesquisa da Ensp/Fiocruz (CAE: 0058.0.031.000-10).

\section{Resultados}

No município de Campina Grande, a amostra total do estudo constituiu-se de 633 pares de mãe e filho, sendo, portanto, superior ao valor calculado para a 
amostra, considerando o desenho do estudo, representando $55,5 \%$ das 1.141 crianças menores de um ano vacinadas no dia da campanha de vacinação.

Como pode ser observado na Tabela 1 , a utilização do serviço público foi de $77 \%$ na assistência pré-natal e de $80,1 \%$ no caso do parto/puerpério. É possível verificar vantagem sistemática na utilização de serviços públicos de saúde durante o pré-natal e o parto/puerpério nas mulheres de menor escolaridade (ensino fundamental incompleto) e nas de famílias com benefício do Programa Bolsa Família. A raça da mulher não foi fator associado ao tipo de serviço usado.

Tabela 1: Distribuição percentual das mães/filhos, segundo características sociodemográficas maternas, de acordo com o tipo de serviço utilizado (público ou privado) na assistência pré-natal e ao parto/pós-parto em Campina Grande, Paraíba, 2010.

\begin{tabular}{|c|c|c|c|c|c|c|c|c|c|c|}
\hline \multirow{3}{*}{$\begin{array}{c}\text { Características } \\
\text { sociodemográficas maternas }\end{array}$} & \multicolumn{5}{|c|}{ Tipo de serviço utilizado no pré-natal* } & \multicolumn{5}{|c|}{ Tipo de serviço utilizado no parto/puerpério ${ }^{+}$} \\
\hline & \multicolumn{2}{|c|}{ Público } & \multicolumn{2}{|c|}{ Privado/Particular } & \multirow[b]{2}{*}{$\mathbf{p}$} & \multicolumn{2}{|c|}{ Público } & \multicolumn{2}{|c|}{ Privado/Particular } & \multirow[b]{2}{*}{$\mathbf{p}$} \\
\hline & $\mathbf{n}$ & $\%$ & $\mathbf{n}$ & $\%$ & & $\mathbf{n}$ & $\%$ & $\mathbf{n}$ & $\%$ & \\
\hline Escolaridade & & & & & 0,001 & & & & & 0,001 \\
\hline Ensino fundamental incompleto & 160 & 90,9 & 16 & 9,1 & & 161 & 90,4 & 17 & 9,6 & \\
\hline Ensino fundamental completo & 28 & 87,5 & 4 & 12,5 & & 27 & 84,4 & 5 & 15,6 & \\
\hline Ensino médio incompleto & 65 & 78,3 & 18 & 21,7 & & 70 & 84,3 & 13 & 15,7 & \\
\hline Ensino médio completo & 171 & 69,2 & 76 & 30,8 & & 189 & 75 & 63 & 25 & \\
\hline Ensino superior & 49 & 62,8 & 29 & 37,2 & & 52 & 65,8 & 27 & 34,2 & \\
\hline Raça & & & & & 0,19 & & & & & 0,31 \\
\hline Branca & 97 & 71,3 & 39 & 28,7 & & 108 & 77,1 & 32 & 22,9 & \\
\hline Parda & 281 & 77,4 & 82 & 22,6 & & 296 & 80,2 & 73 & 19,8 & \\
\hline Negra & 74 & 84,1 & 14 & 15,9 & & 75 & 85,2 & 13 & 14,8 & \\
\hline Indígena/Amarela & 24 & 82,8 & 5 & 17,2 & & 22 & 75,9 & 7 & 24,1 & \\
\hline Chefe da família & & & & & 0,48 & & & & & 0,9 \\
\hline Sim & 138 & 77,5 & 40 & 22,5 & & 146 & 81,1 & 34 & 18,9 & \\
\hline Não & 339 & 77,4 & 99 & 22,6 & & 356 & 79,8 & 90 & 20,2 & \\
\hline Benefício do Programa Bolsa Fam & & & & & 0,001 & & & & & 0,04 \\
\hline $\operatorname{Sim}$ & 141 & 91,6 & 13 & 8,4 & & 139 & 89,7 & & 10,3 & \\
\hline Não & 337 & 72,6 & 127 & 27,4 & & 364 & 77,0 & & 23 & \\
\hline Total & 478 & 77 & 143 & 23 & & 503 & 80,1 & & 19,9 & \\
\hline
\end{tabular}

*n=621 (valores válidos); +n=628 (valores válidos).

\section{Discussão}

Em geral, o setor público constitui a principal forma de utilização dos serviços de saúde da população brasileira, inclusive para os atendimentos de pré-natal e parto/ puerpério, como constatado por meio dos resultados do presente estudo e de outras pesquisas divulgadas anteriormente $e^{9,10,13,14}$. Nesse sentido, cabe ressaltar que esses resultados verificam-se em situaçóes de financiamento minoritário do SUS, em contrapartida a grandes subsídios e maiores coberturas no setor privado, o qual atende populações de melhor situação social, que utilizam, também, os serviços públicos, principalmente aqueles de menor complexidade. Essas circunstâncias explicam as desigualdades na utilização dos serviços de saúde ${ }^{11,13}$. Delimita-se, assim, além de outros inúmeros fatores, a importância da melhoria quantitativa e qualitativa no contexto do SUS.

Apesar das altas taxas de atendimento no SUS, as diferenças relacionadas à utilizaçáo dos serviços de saúde, como no atendimento pré-natal e no parto/puerpério, que foram constatadas nos resultados do presente estudo, convergem com os resultados de pesquisas anteriores, de âmbito nacional e pontual ${ }^{10,13-15}$. Essas diferenças podem gerar desvantagens na assistência à saúde, com piores indicadores nas mulheres atendidas nos serviços públi$\cos ^{14,15}$. Isso implica a necessidade de açóes para melhorar a assistência pública de saúde, pois seu público apresenta maiores dificuldades de acesso aos serviços de saúde - condição associada a fatores como o distanciamento das residências, a falta de orientações e a conformação de redes sociais ${ }^{9,16}$. Por outro lado, sugere-se que a qualidade 
da assistência e a fonte financiadora do serviço podem se dissociar quando do atendimento de clientelas com as mesmas características socioeconômicas ${ }^{14,17}$. Esses fatos indicam a necessidade de refletir sobre a prática profissional e as rotinas dos serviços, independente da fonte que financia o serviço, considerando o cuidado à saúde como direito e exercício de cidadania.

\section{Conclusão}

Os dados apontados no presente estudo sugerem que a maior utilização dos serviços públicos de saúde coexiste com desigualdades sociais entre a população atendida pelo SUS e a população beneficiária de planos de saúde, o que remete à necessidade de ações de melhoria da assistência pré-natal e ao parto e puerpério, sobretudo pelo setor público, caracterizado por atender a população de maior vulnerabilidade.

\section{Agradecimentos}

Ao Departamento de Ciência e Tecnologia do Ministério da Saúde pelo financiamento da pesquisa.

\section{Referências}

1. Melo KL, Vieira BDG, Alves VH, Rodrigues DP, Leão DCMR, Silva LA. O comportamento expresso pela parturiente durante o trabalho de parto: reflexos da assistência do pré-natal. J Res Fundam Care. 2013;6(3):1007-20.

2. Brasil. Ministério da Saúde. Secretaria de Atenção à Saúde. Departamento de Açôes Programáticas Estratégicas. Portaria $\mathrm{n}^{\mathrm{o}} 1.459$, de 24 de junho de 2011. Institui, no âmbito do Sistema Único de Saúde - SUS - a Rede Cegonha. Brasília: Ministério da Saúde; 2011.

3. Cabral FB, Hirt LM, Van der Sand ICP. Atendimento pré-natal na ótica de puérperas: da medicalização à fragmentação do cuidado. Rev Esc Enferm USP. 2013;47(2):281-7.

4. Cardoso LSM, Mendes LL, Velasquez-Melendez G. Diferenças na atenção pré-natal nas áreas urbanas e rurais do Brasil: estudo transversal de base populacional. REME Rev Min Enferm. 2013;17(1):85-92.

5. Brasil. Ministério da Saúde. Secretaria Executiva. Programa de Humanização do Parto e Nascimento. Brasília: Ministério da Saúde; 2002.
6. Leal MC, Theme-Filha MM, Moura EC, Cecatti JG, Santos LMP. Atenção ao pré-natal e parto em mulheres usuárias do sistema público de saúde, SUS, residentes na Amazônia Legal e no Nordeste, Brasil 2010. Rev Bras Saúde Matern Infant. 2015;15(1):91-104.

7. Comissão Nacional sobre Determinantes Sociais da Saúde. As causas sociais das iniqüidades em saúde no Brasil. Rio de Janeiro: Fiocruz; 2008.

8. Alves GJ, Leandro SS. Abordagem a populaçôes em situaçâo de vulnerabilidade na Atenção Primária à Saúde. In: Kalinowski CE, Figueiredo KC, Fonseca RMGS, organizadores. PROENF Programa de Atualização em Enfermagem: Atenção Primária e Saúde da Família: Ciclo 2. Porto Alegre: Artmed Panamericana; 2014. p. 93-124. (Sistema de Educação Continuada a Distância, v. 4).

9. Paris GF, Pelloso SM, Martins PM. Qualidade da assistência pré-natal nos serviços públicos e privados. Rev Bras Ginecol Obstet. 2013;35(10):447-52.

10. Cesar JA, Mano PS, Carlotto K, Gonzalez-Chica DA, Mendoza-Sassi RA. Público versus privado: avaliando a assistência à gestação e ao parto no extremo sul do Brasil. Rev Bras Saúde Matern Infant. 2011;11(3):257-63.

11. Santos IS, Ugá MAD, Porto SM. O mix público-privado no Sistema de Saúde Brasileiro: financiamento, oferta e utilização de serviços de saúde. Ciênc Saúde Colet. 2008;13(5):1431-40.

12. Brasil. Ministério da Saúde. Secretaria de Ciência, Tecnologia e Insumos Estratégicos. Departamento de Ciência e Tecnologia. Avaliação da atenção ao pré-natal, ao parto e aos menores de um ano na Amazônia Legal e no Nordeste, Brasil, 2010. Brasília; Ministério da Saúde; 2013.

13. Porto SM, Ugá MAD, Moreira RS. Uma análise da utilização de serviços de saúde por sistema de financiamento: Brasil 1998-2008. Ciênc Saúde Colet. 2011;16(9):3795-806.

14. Domingues RMSM, Viellas EF, Dias MAB, Torres JA, Theme-Filha MM, Gama SGN, et al. Adequação da assistência pré-natal segundo as características maternas no Brasil. Rev Panam Salud Publica. 2015;37(3):140-7.

15. Cesar JA, Sutil AT, Santos GB, Cunha CF, Mendoza-Sassi RA. Assistência pré-natal nos serviços públicos e privados de saúde: estudo transversal de base populacional em Rio Grande, Rio Grande do Sul, Brasil. Cad Saúde Pública. 2012;28(11):2106-14.

16. Osorio AM, Tovar LM, Rathmann K. Individual and local level factors and antenatal care use in Colombia: a multilevel analysis. Cad Saúde Pública. 2014;30(5):1079-92.

17. García FM. Los determinantes de la calidad de la atención en salud: el caso del control prenatal. Coyunt Econ. 2009;34(2):61-97.

\section{Como citar este artigo:}

Pedraza DF, Araujo E. Assistência materno-infantil, tipo de serviço utilizado e características sociodemográficas em cidade do interior da Paraíba. Rev. Aten. Saúde. 2017;15(52):11-14. 